\title{
Population-level interventions to reduce the development and transmission of community-associated antimicrobial resistance: A perspective from the National Collaborating Centre for Infectious Diseases
}

\author{
Margaret Fast MD, Kelly Bunzeluk MSc
}

\begin{abstract}
A ntimicrobial resistance (AMR) in bacteria is the end result of a multitude of factors. Some of the key factors beyond innate resistance include antimicrobial selective pressure (1-5), acquisition of a foreign genetic resistance element(s) (1-5), clonal dissemination (1) and new mutations $(2,3,5)$; factors vary for different species and geographical locations. Increased global antimicrobial use is the foremost reason for the spread of AMR in the community setting (1). Social networks of individuals (households, schools and child care facilities) have served both as a reservoir for these bacteria and as a common route for their transmission. Similarly, hospitals, nursing homes and long-term care facilities have also served as reservoirs for antibiotic-resistant organisms, and the discharge of patients from these facilities contributes to the spread of resistance within communities. In addition, the use of antimicrobials in food animals has been an important contributing cause (1).

In the preantibiotic era, the mortality of patients infected with Staphylococcus aureus bacteremia exceeded $80 \%$. Although the introduction of penicillin in the early 1940s significantly improved the prognosis of patients with staphylococcal infection, penicillin-resistant staphylococci were recognized within two years of its use $(4,6)$. More than $80 \%$ of both communityand hospital-acquired staphylococcal isolates were resistant to penicillin by the late 1960s. This transfer of resistance, from the hospital setting to communities, is a well-established pattern that recurs with each new wave of AMR (6). For example, the introduction of methicillin in 1961 was quickly followed by reports of methicillin-resistant isolates in hospitals, and is now seen regularly in community-based infections (6).

Health care-associated strains of methicillin-resistant $S$ aureus (HA-MRSA) have been known to cause a wide variety of infections (eg, wound infections, catheter-associated bacteremias or prosthesis infections). Community-acquired MRSA (CA-MRSA) has emerged as the causative agent of serious individual cases and outbreaks of skin and soft tissue infections and sepsis. Infections have been noted among those without the usual HA-MRSA risk factors (history of hospitalization or other institutionalization, antibiotic use, dialysis and chronic wounds), and outbreaks have been reported in well-defined epidemiological groups (children in child care facilities, athletes, military recruits and prison inmates) (1,7). CA-MRSA is distinguished
\end{abstract}

from HA-MRSA, in part, by the type of staphylococcal chromosomal cassette - a mobile chromosomal element that carries the methicillin resistance gene mec. However, there is a progressive blurring of the two categories. HA-MRSA is also disseminated in the community (for example, MRSA has been transmitted from hospital patients to their household contacts), and CA-MRSA has entered health care settings and caused outbreaks in hospitals (1).

Vancomycin-resistant enterococci, extended-spectrum beta-lactamase-producing organisms that are resistant to cephalosporins and monobactams, and penicillin-resistant Streptococcus pneumoniae have also evolved through a combination of acquired foreign genetic material, antibiotic selective pressure and clonal dissemination (1). Vancomycin-resistant enterococci have been associated with the use of growth promoters in food animals, especially in Europe (1), and extendedspectrum beta-lactamase-producing Gram negatives, especially Klebsiella and Escherichia coli, have been increasingly noted as a source of community-acquired infections (8). Similarly, penicillinresistant $S$ pneumoniae, a common cause of otitis media, sinusitis, community-acquired pneumonia, meningitis and bacteremia, has emerged in the past few decades - first identified more than 20 years after the introduction of penicillin $(1,8)$.

Although the ultimate impact of AMR in the community is still uncertain, reducing the development and limiting the spread of resistant organisms should be a public health priority. The two most urgent proven strategies to effect this goal are to limit antimicrobial use to prevent selective pressure, and to enhance infection prevention and control practices, including basic hand hygiene, to prevent clonal dissemination $(1,2,9,10)$.

Several national and regional groups in Canada are currently investigating AMR issues, and numerous policy and practice guidance documents have been produced. Despite these efforts, public health leaders and practitioners across Canada have argued that knowledge and practice gaps still exist on the issue of AMR, particularly in community settings.

With the assistance of an expert advisory group, the National Collaborating Centre for Infectious Diseases (NCCID) identified projects that could help reduce the burden of community-acquired AMR in Canada. The goal of one project was to describe population-level interventions to reduce the development and transmission of community-associated

National Collaborating Centre for Infectious Diseases, Winnipeg, Manitoba

Correspondence: Dr Margaret Fast, National Collaborating Centre for Infectious Diseases, 413-445 Ellice Avenue, Winnipeg, Manitoba R3B 3P5. Telephone 204-943-0051, fax 204-946-0927, e-mail mfast@icid.com

Reprints: National Collaborating Centre for Infectious Diseases, 413-445 Ellice Avenue, Winnipeg, Manitoba R3B 3P5.

Telephone 204-943-0051, fax 204-946-0927, e-mail nccid@icid.com 
AMR. NCCID issued a call for proposals in the summer of 2008, and in the fall of 2008, a selection process was undertaken that included peer review by independent, external experts. Three proposals were selected for NCCID funding:

- Antimicrobial use and resistance in pigs and chickens: A review of the science, policy and control practices from farm to slaughter - Leigh B Rosengren, Sheryl P Gow and J Scott Weese.

- A review of alternative practices to antimicrobial use for disease control in the commercial feedlot - Carl S Ribble, Tyler Stitt, S Iwasawa, Lorraine Toews and Craig Stephen.

- Strategies to control community-associated antimicrobial resistance among enteric bacteria and methicillin-resistant Staphylococcus aureus in Canada - Jeff Wilson, John Conly, Thomas Wong, Gayatri Jayaraman, Jan Sargeant, Andrew Papadopoulos, Virginia Young, Melanie Quist-Moyer and Sharon Bauer.

Drafts of the comprehensive reviews were read by a minimum of five independent reviewers. Feedback was compiled and provided to the authors for incorporation.

The authors presented their general findings at a communityacquired AMR consultation hosted by the NCCID in February 2010. The consultation, which included 50 experts from human, animal and environmental health fields, allowed the reviews to be further refined. Proceedings from the consultation are available on the NCCID website (www.nccid.ca/en/ caamr-consultation-feb10).

The subsequent pages present the executive summaries of the three reviews (the full comprehensive reviews are available online at http://nccid.ca/en/amr-reviews). The first two reviews focus on antimicrobial use and resistance in animals raised for food, namely pigs and chickens (11) and cattle (12). The third review discusses strategies to control community-acquired AMR among human enteric bacteria and MRSA (7). It is hoped that these papers will lead to further discussion and research into the most effective strategies to reduce the development and spread of AMR.

\section{ABOUT NCCID}

NCCID is one of six national collaborating centres for public health funded by the Public Health Agency of Canada. NCCID's mission is to serve as a bridge between research and knowledge and the practical needs of front-line public health practitioners. NCCID acknowledges the complexities of public health problems and promotes the use of evidence to implement infectious disease prevention initiatives. This is accomplished through the following ways:

- Identification of knowledge gaps in research and practice;

- Knowledge synthesis, translation, and exchange to incorporate evidence from research and experience into policy and practice; and

- Network development to support the use of evidence in public health decision making.

NOTE: The National Collaborating Centre for Infectious Diseases is hosted by the International Centre for Infectious Disease - a nonprofit organization located in Winnipeg, Manitoba.

\section{REFERENCES}

1. Furuya EY, Lowy FD. Antimicrobial-resistant bacteria in the community setting. Nat Rev Microbiol 2006;4:36-45.

2. Tenover FC. Mechanisms of antimicrobial resistance in bacteria. Am J Med 2006;119(6 Suppl 1):S3-10.

3. Davies J. Inactivation of antibiotics and the dissemination of resistance genes. Science 1994;264:275-382.

4. Walsh C. Molecular mechanisms that confer antibacterial drug resistance. Nature 2000;406:775-81.

5. Swartz MN. Use of antimicrobial agents and drug resistance. N Engl J Med 1997;337:491-2.

6. Lowy F. Antimicrobial resistance: The example of Staphylococcus aureus. J Clin Invest 2003;111:1265-73.

7. Wilson J, Conly J, Wong T, et al. Strategies to control communityassociated antimicrobial resistance among enteric bacteria and methicillin-resistant Staphylococcus aureus in Canada - executive summary. Can J Infect Dis Med Microbiol 2010;21:133-4.

8. Senekal M. Current resistance issues in antimicrobial therapy. CME 2010;28:54-7.

9. Nicolle L, Conly JM, MacDonald N. Embracing ecology to limit antimicrobial resistance. CMAJ 2009;180:371-2.

10. Patrick DM, Hutchinson J. Antibiotic use and population ecology: How you can reduce your "resistance footprint." CMAJ 2009; 180:416-21.

11. Rosengren LB, Gow SP, Weese JS. Antimicrobial use and resistance in pigs and chickens: A review of the science, policy, and control practices from farm to slaughter - executive summary. Can J Infect Dis Med Microbiol 2010;21:123-4.

12. Ribble CS, Stitt T, Iwasawa S, Toews L, Stephen C. A review of alternative practices to antimicrobial use for disease control in the commercial feedlot - executive summary. Can J Infect Dis Med Microbiol 2010;21:128-9. 


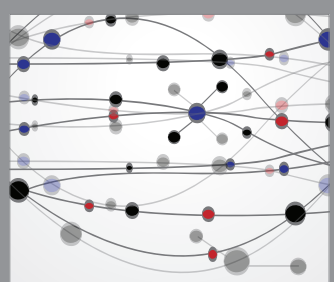

The Scientific World Journal
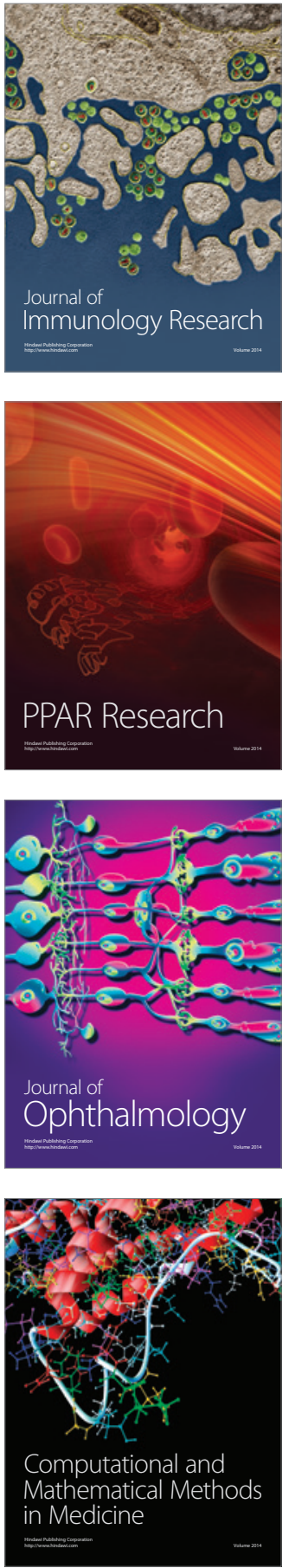

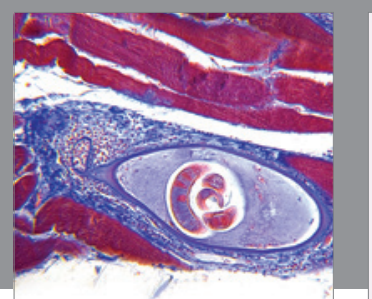

Gastroenterology Research and Practice

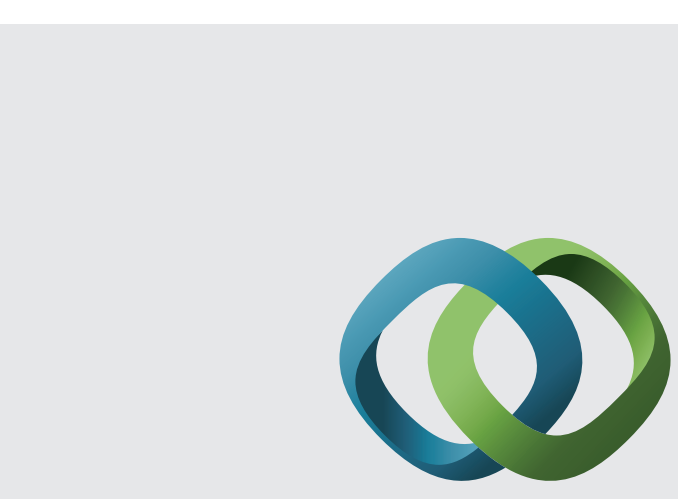

\section{Hindawi}

Submit your manuscripts at

http://www.hindawi.com
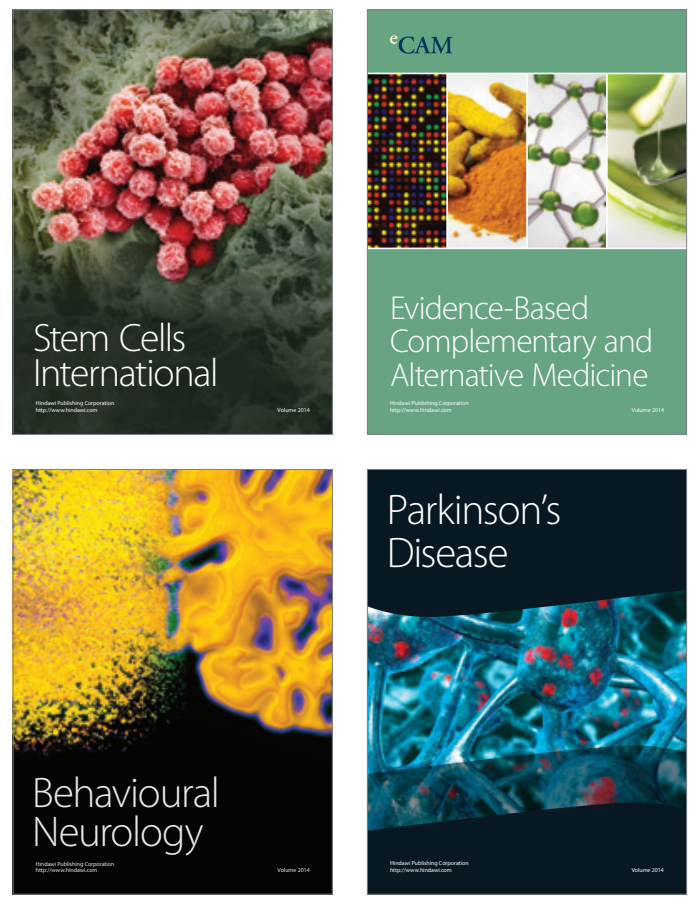
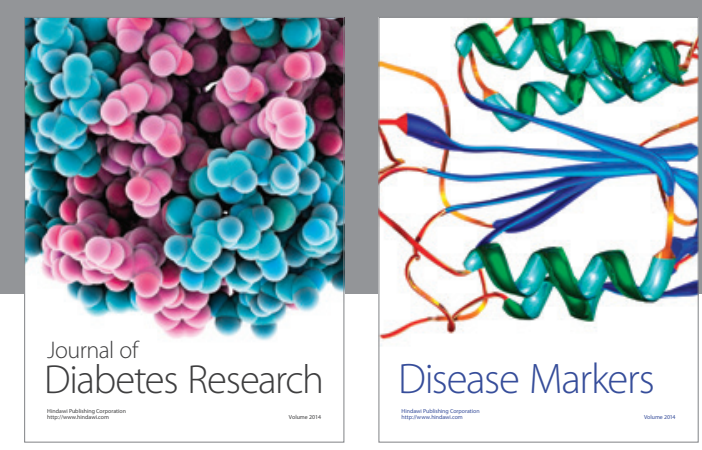

Disease Markers
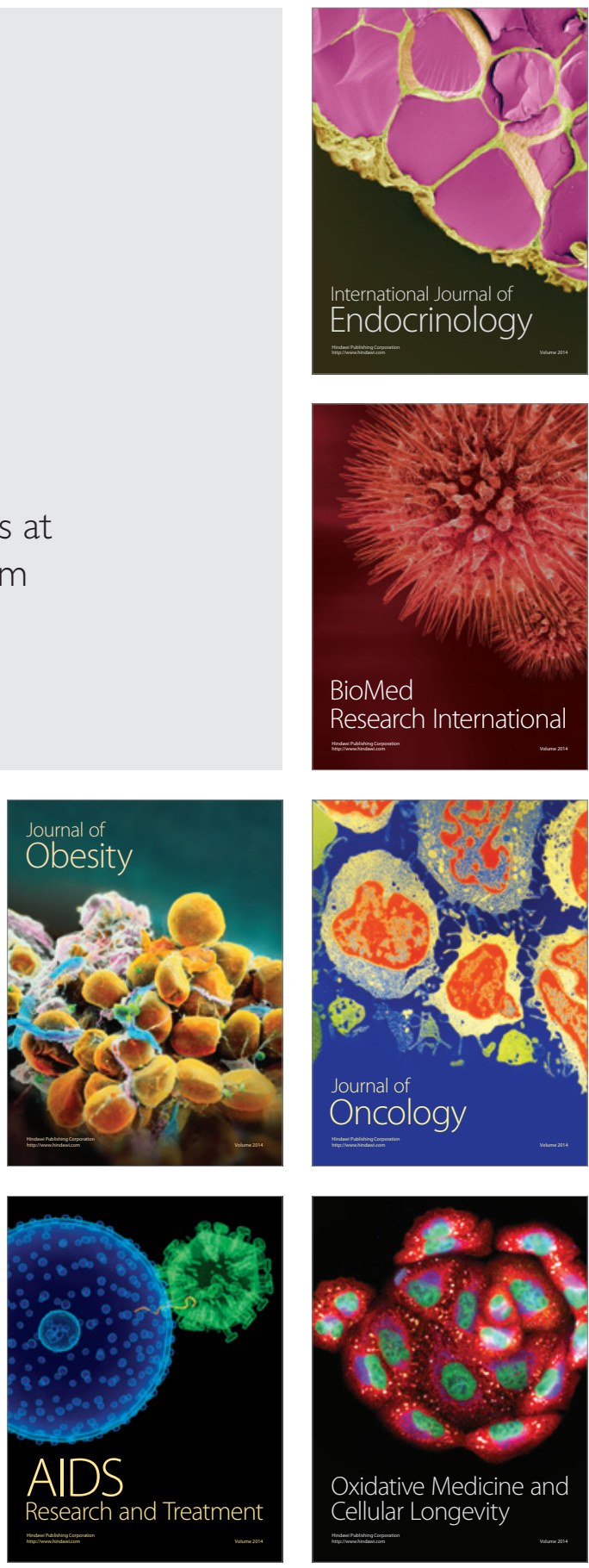